\title{
AUX MARCHES DU PALAIS: \\ LES ACCÈS AU PALATIUM DE 54 À 70 \\ Par \\ YVES PERRIN
}

\section{Introduction}

Les travaux archéologiques récents ont amené un important renouvellement des connaissances sur le Palatium néronien et ses abords. Dans une démarche historique fondée sur la confrontation des sources écrites et archéologiques, ma contribution portera sur un seul point: les accès au Palatium entre 54 et 70 . Les événements souvent dramatiques qui prennent place sur le Palatin et à ses abords pendant cette période amènent les auteurs à évoquer le décor dans lequel ils se déroulent. Alors que les termes ianua et porta reviennent constamment à propos du Palatin archaïque, républicain et augustéen, ils n'apparaissent que de manière statistiquement négligeable pour la période 54 - 70. Les auteurs emploient les termes fores (thuriai) Palatii et aditus domus auxquels ils associent des gradus. Ces termes, je vais essayer de le montrer, renvoient à des réalités distinctes dont les données archéologiques permettent d'envisager la topographie et l'architecture.

L'entreprise n'est pas aisée: en dépit des progrès de la connaissance archéologique, la question récurrente des limites des domus néroniennes et donc de leurs accès reste posée. Nos informations sont inégalement réparties dans le temps et singulièrement plus riches pour les années 68-70 que pour le règne de Néron lui-même, mais ce n'est pas un handicap puisque les guerres civiles se déroulent dans le décor néronien. Les termes fores, aditus, gradus autour desquels je me propose d'organiser l'analyse sont neutres, les réalités qu'ils désignent plurielles et susceptibles d'avoir topographiquement et architecturalement changé au cours des décennies 54-70 marquées par les réaménagements, voire les bouleversements engendrés par la création de la domus transitoria, de la domus tiberiana, puis l'incendie de 64 et la domus aurea. En dépit de ces limites, l'enrichissement des données permet des considérations qui auraient été impossibles il y a une vingtaine d'années sur les modalités politiques et fonctionnelles, spatiales et architecturales de la formation du palais impérial et ses relations avec la ville entre 54 et 70 et sur la mise en scène du pouvoir.

\section{Les données textuelles et lexicologiques.}

En 54, Claude est empoisonné au Palatium, in domestico convivio, et meurt 
dans le cubiculum de sa domus. Il est mourant, mort déjà peut-être. Agrippine en conserve le secret dans l'attente du moment le plus favorable pour faire proclamer son fils. Elle retient Britannicus dans le cubiculum et empêche quiconque d'approcher le vieux prince en faisant fermer les aditus par la custodia. Le moment venu, les fores Palatii s'ouvrent, Néron rencontre les excubitores et est salué imperator pro gradibus Palatii. ${ }^{1}$ Pour la période 5468 , les occurences sont rares. Suétone évoque les cruautés commises par Néron sur le Palatium au cours de la première partie du règne et enchaîne en ajoutant qu'il se conduit avec non moins de cruauté hors de sa maison et envers les étrangers, foris et in exteros. ${ }^{2}$ Dans le cadre de la domus aurea, les termes foris, aditus, gradus ne sont jamais utilisés. En revanche la littérature latine contemporaine multiplie les occurences du terme aula pour désigner un complexe palatial fermé par des fores, notamment le palais d'Alexandrie chez Lucain. ${ }^{3}$ Or, j'y reviendrai, les complexes palatiaux qu'évoquent Lucain et Sénèque (et l'Octavie) en les désignant comme des aulae ne sont pas sans rapport avec les résidences néroniennes qu'ils fréquentent.

La période des guerres civiles de 68-70 fournit le plus grand nombre d'occurences: au cours de la lutte entre Galba et Othon, chevaliers et sénateurs forcent les fores Palatii pour rencontrer Galba. Ayant appris qu'Othon a été proclamé, celui-ci laisse à Pison le soin de haranguer la cohors quae in Palatio stationem agebat, ce que Pison fait pro gradibus domus. ${ }^{4}$ A la fin du discours, les speculatores se dispersent, mais le reste de la cohorte a un comportement ambigu. La populace envahit alors le Palatium. Inquiets, les proches de Galba sont divisés. Les uns jugent préférable qu'il reste intra domus et en barricade les aditus. D'autres lui conseillent de ne pas s'enfermer chez lui comme s'il était décidé à soutenir un siège (ianuae limine tenus domum cludit). ${ }^{5}$ Pendant le conflit Othon Vitellius, l'assaut des vitelliens ne se brise pas contre les fores Palatii; ils parviennent jusqu'à la salle où Othon offre un festin aux primores de Rome. ${ }^{6}$ C'est pro gradibus Palatii que Vitellius s'adresse à ses partisans pour leur

\footnotetext{
${ }^{1}$ Tacite, Annales 11.37 et 12.68-69; cf. Suétone, Claude $44-45$ et Néron 8.

${ }^{2}$ Suétone, Néron 36.

${ }^{3}$ J'ai compté 35 occurences du mot aula dans l'Octavie, 40 chez Lucain, 30 chez Sénèque. Pour les fores de l' aula, voir Pharsale 5.440 (cf. aussi 2.165 ; 10.11, 72, 112, 160, 422, 485 etc.); Octavie 275, $624,689,698-699,780,790,851,892$ etc. Cf. infra n. 36.

${ }^{4}$ Tacite, Histoires 1.27-33, 35 ; Suétone, Othon 6 ; Plutarque, Galba 31.

${ }^{5}$ Tacite, Histoires 1.31-32.

${ }^{6}$ Tacite, Histoires 1.82 ; Dion Cassius 64.9.
} 
annoncer qu'il renonce au pouvoir, ensuite pour tenter de sauver Sabinus. ${ }^{7}$ Lors de l'épisode final de la lutte contre les Flaviens, il se cache dans la cellula ianitoris aux fores desquelles il attache le chien de garde. ${ }^{8}$ La paix rétablie, Vespasien laisse symboliquement les thuras du Palatium ouvertes. A la veille de son triomphe, les troupes ne gagnent pas "les thuras du palais d'en haut", mais le sanctuaire d'Isis.'

Quelles leçons tirer de ce recensement? Les auteurs distinguent des fores ou thuras Palatii associées à des gradus Palatii et des aditus domus associés à des gradus domus. ${ }^{10}$ Les fores sont de véritables portes (au sens vitruvien: à deux battants ${ }^{11}$ ) qui s'ouvrent lors de l'avènement de Néron et que forcent les sénateurs sous Galba, les soldats sous Othon. Ce sont les portes non d'une domus, mais du Palatium, de l'aire princière du Palatin, de l'aula. L'équivalent grec - thurai - ne laisse aucun doute: ce sont les portes d'une aire palatiale. ${ }^{12}$ Bien que les textes n'associent pas explicitement gradus Palatii et fores Palatii, tout suggère qu' ils sont voisins. Ils sont gardés par les prétoriens de la cohorte. C'est en 54 que sont mentionnés pour la première fois des fores et gradus Palatii, sous Trajan qu'ils apparaissent pour la dernière fois (Plotine se retourne après avoir gravi les gradus pour s'adresser à la foule ${ }^{13}$ ).

Dans son acceptation architecturale, imprégnée de références à la vie sociale, aditus désigne le seuil de la domus, l'accès au vestibule, l'accès à la cella dans les édifices sacrés, et l'admission à le franchir, autrement dit l'accès auprès du maître, le prince dans le cas de la résidence impériale. ${ }^{14}$ Dans les occurences recensées, il désigne les accès de domus situées sur le Palatin. Les aditus sont gardés par la custodia et/ou les speculatores. C'est en 68 que sont mentionnés pour la première fois les gradus domus.

Dans les passages recensés, gradus est toujours au pluriel (pro gradibus). Le terme désigne donc des gradins, mais leur nature est imprécisée: il peut s'agir de volées d'escaliers, de quelques marches ou d'une barrière de

\footnotetext{
${ }^{7}$ Tacite, Histoires 3.74, Suétone, Vitellius 15.

${ }^{8}$ Suétone, Vitellius 16; cf. Tacite, Histoires 3.84 .

${ }^{9}$ Flavius Josèphe, La guerre des Juifs 7.5.123.

${ }^{10}$ Sont attestés des passages discrets du côté du Vélabre, dans la domus tiberiana (Suétone, Claude 18; Othon 6; Vitellius 16; Tacite, Histoires 1.27-33, 35; 3.71, 84; Plutarque, Galba 24). L'archéologie confirme l'existence de corridors dans cette zone (M.A. Tomei, Archeologia Laziale 8 [1987], 71).

${ }^{11}$ Vitruve, De architectura 5.6, 4, 7.

${ }^{12}$ Aula retrouve le sens qui était le sien à l'époque hellénistique, chez Théocrite par exemple où aulè désigne le palais royal. Cf. Xénophon, Anabase 1.9.3; Aristote, Politique 5.11.6.

${ }^{13}$ Tacite, Annales 12.69 ; Suétone, Néron 8 ; Dion Cassius 68.5.5.

${ }^{14}$ Vitruve 1.2.6. Cf Cicéron, De l'orateur 3.133. Poppée est admise près du prince: accepto aditu. Le chevalier Bassus achète une audience: principis aditum emercatus (Tacite, Annales 12.68-69; 13.46; Suétone, Claude 44-45 et Néron 8).
} 
contrôle. Lorsque Pline loue Nerva pour avoir ouvert libéralement le Palatin, il écrit que l'arx de Domitien est désormais une aedes où sont supprimés "les barrières, les échelons de contrôle humiliants ... après avoir franchi mille portes, on ne trouve pas au-delà toujours de nouveaux et redoutables obstacles" (nullae obices, nulli contumeliarum gradus superatisque iam mille liminibus ultra semper aliqua dura et obstantia). ${ }^{15}$

$\mathrm{Au}$ terme de cette brève analyse, il apparaît que fores et aditus sont deux réalités distinctes et non interchangeables. Il n'est jamais question des fores domus ou des aditus Palatii. Confirment la distinction les corps de garde qui leur sont spécifiquement affectés. Les auteurs distinguent et en général opposent la custodia et les speculatores, des hommes de confiance qui veillent sur les aditus et vraisemblablement les passages secrets, et la cohorte des prétoriens qui gardent les fores du Palatium et sont peu sûrs.

Fondée étymologiquement - foris désigne la porte qui clôt non la maison, mais l'enclos - et adverbialement - foris signifie dehors et s'oppose à domus $^{16}$ - la distinction est couramment attestée. Lorsqu'ils assaillent le Capitole, les vitelliens forcent d'abord les primas Capitolinae arcis fores, la première ligne de défense, puis les aditus, une deuxième ligne correspondant à l'area capitolina ${ }^{17}$ Lorsque Enée consulte la Sibylle, c'est ante fores que le dieu en prend possession; c'est par l'aditus qu'il pénètre dans la salle de consultation. ${ }^{18}$ C'est cette distinction que suggère Pline le Jeune lorsqu'il loue Nerva d'avoir supprimé la multiplicité des contrôles. La polyvalence des termes fores et aditus et la pluralité des entrées qu'ils désignent rendent leur identification délicate sur le terrain, d'autant plus que les sources assimilent souvent l'aire palatiale et des domus qui s'y trouvent comme l'illustre un certain nombre de formules littéraires et épigraphiques, notamment domus palatina. ${ }^{19}$ Néanmoins, si les deux réalités se recoupent, voire s'identifient, tous les auteurs distinguent l'aire palatiale (Palatium, aula, basileion chez Josèphe) close par des fores et les domus qui s'y trouvent avec leurs aditus.

\footnotetext{
${ }^{15}$ Pline le Jeune, Panégyrique 47, traduction M. Durry.

${ }^{16}$ Tacite emploie fores pour les entrées d'un jardin (Annales 13.58 par exemple). Pour l'étymologie, voir Ernout \& Meillet, Dictionnaire étymologique, s.v. foris, 246.

${ }^{17}$ D. Filippi, 'L'arx capitolina e le primae Capitolinae arcis fores di Tacito', Bullettino della Commissione Archeologica Comunale in Roma 49, ns 8, $73 \mathrm{ff}$.

${ }^{18}$ Virgile, Enéide $6.97 ; 6.43,98$. Vitruve 4.3, 4, 11, emploie fores pour désigner l'accès à la cella à travers le pronaos.

${ }^{19}$ Voir M. Royo, Domus Imperatoriae. Topographie, formation et imaginaire des palais impériaux $d u$ Palatin. Bibl. des Écoles Françaises d' Athènes et de Rome 303 (Rome, 1999), 170 ff. et 237-238); c'est au fastigium de la domus palatina que Claude suspend sa couronne navale (Suétone, Claude 17). Tacite assimile à plusieurs reprises domus du prince et Palatium (Annales 12.5 et 68-69 par exemple).
} 


\section{Les accès au Palatium et à ses domus de 54 à 64: la domus transitoria $(\text { plan I })^{20}$}

Lorsque Néron arrive au pouvoir en 54, il hérite de la résidence impériale telle que ses prédécesseurs l'ont constituée. Elle réunit au sein d'un seul ensemble plusieurs demeures, notamment celle d'Auguste - celle des Iulii - et celle de Claude, héritier de celles des Claudii, Tibère, Germanicus, Caligula .

Le site des résidences des Claudii et de Claude en particulier est débattu. ${ }^{21}$ En l'état des connaissances, la proposition la plus simple est qu'il corresponde à l'aire septentrionale du site de la domus tiberiana. L'explication la plus plausible qu'on puisse avancer du curieux nom de domus tiberiana qui n'apparaît qu'en 69 est d'y voir le souvenir de la domus de Tibère, voisine de celles de Germanicus et Caligula qui occupe l'angle nord-ouest du site. ${ }^{22}$ Les luxueuses salles qui gisent sous l'angle nord-ouest du podium de la domus tiberiana (S du plan I) n'ont à ma connaissance jamais été prises en considération dans le débat. Elles appartiennent à un ensemble plus vaste, dont on ignore l'extension, mais dont on il est légitime de penser qu'il atteint l' 'area palatina' à l'Est et le 'clivus Victoriae' au nord (sont attestés des escaliers vers le clivus); de construction tardorépublicaine, ces salles ont connu des réaménagements (le dernier peutêtre lié à un incendie dont témoignent d'éventuelles traces sur certains fragments de marbre) et le style de la décoration musivale est claudien. ${ }^{23}$ Or nous savons que, en 41 , Claude hérite de la résidence de son prédécesseur et que celle-ci brûle. On peut donc avancer que ces salles appartiennent à un quartier de la domus des Claudii détruit en 41, puis rénové par Claude et ensuite enfoui sous le podium de la domus tiberiana. $\mathrm{Si}$ on accepte ces propositions, l'ensemble résidentiel claudien a une façade tournée vers l' 'area palatina', une autre vers le 'clivus Victoriae'.

Les indications que fournissent Josèphe et Tacite sur le cadre dans

\footnotetext{
${ }^{20}$ J'utilise par commodité les termes area palatina, clivus palatinus et clivus Victoriae dans l' acceptation que leur a donnée l'historiographie, qui est inexacte. Aussi les ai-je placés entre guillemets.

${ }^{21}$ Voir Royo 1999, op cit. (n. 19), 83 et 273 ff.

${ }^{22}$ Cf. W. Eck, 'Domus: Ti. Claudius Nero', dans LTUR 2, 83 et H. Hurst, Domus Gai, dans LTUR 2, 106-108.

${ }^{23}$ Les mosaïques orneraient un triclinium (A.M. Tomei, Archeologia Laziale 8 [1987], 72). M. de Vos, 'Tre ambianti com mosaico parietale sotto gli Horti Farnesiani sul Palatino', La mosaïque grécoromaine 4 (Paris 1984), 83-86, date la décoration entre la fin du règne de Tibère et 64 . La technique musivale et les cabochons de la voûte, semblables à ceux du nymphée dit de la domus transitoria, dont je retiens la datation claudienne (cf. V. Strocka, 'Die römische Wandmalerei von Tiberius bis Nero', Pictores per provincias, Cahiers d'Archéologie romande 43 [1987], 29-44), incitent à retenir une datation claudienne.
} 
lequel les soldats proclament Claude en 41 et Néron en 54 semblent conforter l'identification. Le récit que fait Josèphe de la proclamation de Claude n'est pas linéaire. Du paragraphe 104 au paragraphe 165, il évoque la première séquence: Caligula est assassiné dans le basileion où Claude se trouve également, mais en un autre endroit (104-106); les assassins s'enfuient dans l'oikia de Germanicus, proche de celle de Caligula (117). Les Germains les recherchent, la panique règne. Telle est la situation lorsque Claude est enlevé de sa demeure (ek tès oikias) par les soldats qui ont décidé de le proclamer (162-165). Josèphe interrompt alors son récit. Il y revient au paragraphe 212. Après avoir rappelé le climat de panique, il enchaîne: Claude se cache sur une terrasse où il est découvert par le prétorien Gratus qui lui apprend la décision des soldats de le proclamer. Gratus l'entraîne sur l'euruchoria du Palatin où les prétoriens le proclament près d'un demosion (217-218). Claude est donc caché dans sa domus (ou une domus des Claudii) qu'on ne voit pas où situer sinon sur l'aire septentrionale du Palatium. L'euruchoria où les soldats l'entraînent correspond à l' 'area palatina', seule aire où peut logiquement se regrouper un grand nombre des soldats de la garde ${ }^{24}$ Le demosion près duquel se déroule 1' acclamatio est une 'salle publique d'audience' plutôt que 'la partie publique du palais' ou un trésor. ${ }^{25}$ Ce pourrait être l'aula qui, au sud de l'area, occupe le site de l'aula regia domitianéenne, que la recherche n'a jamais prise en compte. ${ }^{26}$

Passons à la proclamation de Néron selon Tacite. Claude meurt dans sa domus, Agrippine y retient Britannicus. Le moment venu, les fores Palatii s'ouvrent, Néron rencontre la cohorte et est salué imperator près des fores Palatii. Tacite ajoute: au moment de la proclamation, certains soldats

\footnotetext{
${ }^{24} \mathrm{~L}$ ' identification de l' 'area palatina' et de l' euruchoria est reprise par T. Wiseman, Flavius Josephus. Death of an Emperor. Exeter Studies in History 30 (Exeter 1991). Elle est rejetée par M. Royo 1999, op. cit. (n. 19), 275-288, qui situe l'euruchoria près de la domus d'Auguste. La principale objection à l'identification réside dans la relation établie par Josèphe entre l'euruchoria et le lieu de la fondation de Rome. Mais des arguments existent pour répondre à l'objection (voir T. Wiseman, 'The Public Image of Aristocratic and Imperial Houses', dans L'Urbs. Espace urbain et histoire. Coll. de l'École Française de Rome 98 (Rome 1987), 403-404 et P. Tassini, 'Una memoria di Remo alle pendici del Palatino', Archeologia Classica 45 (1993), 333-350.

${ }^{25}$ Dans l'édition Loeb, L. Feldman traduit demosion par "treasury", qui ne peut convenir comme le montre M. Royo 1999, op. cit.(n. 19), 275-288. Mais je ne retiens pas la traduction " partie publique du palais" proposée par M. Royo. Le demosion qu'évoque Dion Cassius 54.27 pour un épisode de la période augustéenne est une salle.

${ }^{26}$ L'aula domitianéenne succède à deux constructions. C.F. Giuliani, 'Architettura delle residenze imperiali', dans W.Haase \& H. Temporini, Aufstieg und Niedergang der Römischen Welt II 12.1 (Berlin/New York 1982), 250 date sans argumenter les deux premières phases de Néron et/ou de Vespasien; il n'y a aucune raison de ne pas suivre la chronologie de l'inventeur, Boni, qui date la première construction de Tibère.
} 
hésitent, regardent derrière eux en demandant où est Britannicus (Britannicus dubitavisse quosdam ferunt, respectantis rogitantisque ubi Britannicus esset) ${ }^{27} \mathrm{Je}$ comprends que ces soldats en faction aux fores regardent la façade de la domus où est enfermé Britannicus, autrement dit celle où meurt Claude. ${ }^{28}$

Les proclamations de Claude et de Néron se déroulent donc dans un décor où apparait la domus des Claudii (ou de Claude) qu'il semble logique de situer sur l'aire septentrionale du Palatin. Tout incite à penser que cette résidence a une façade tournée vers l' 'area palatina' et une autre vers le 'clivus Victoriae'. C'est sur l'une d'elles (et non sur la façade de la domus d'Auguste) qu'il faut imaginer le fastigium et la couronne célébrant sa conquête de la Bretagne. ${ }^{29}$

D'où la garde peut-elle, en 54, apercevoir cette façade? Soit depuis le 'clivus Victoriae', soit depuis le 'clivus palatinus'. Aucune des deux possibilités ne peut être exclue. Que voit-on depuis la partie haute du 'clivus palatinus' ? A l'Est, sur le site de la Vigna Barberini, s'élève une (ou des domus) dont les archéologues français pensent qu'elle était luxueuse mais médiocrement construite comme s'il s'agissait d'un édifice conçu pour la mise en scène ${ }^{30}$ Au sud s'étend l' 'area palatina', qui est la plus susceptible d'accueillir l'attroupement des prétoriens et à travers laquelle les soldats peuvent voir la façade de la domus de Claude. Il semble donc plausible de placer la proclamation de Néron près des fores du 'clivus palatinus', sans pouvoir exclure cependant le 'clivus Victoriae' dont l'environnement est mal connu. ${ }^{31}$ Mais on n'est pas en mesure de préciser les modalités du passage entre aire publique et aire impériale. Certaines des domus voisines appartiennent à l'empereur, d'autres à des proches (Caecina Largus) et à des particuliers. Quant aux gradus Palatii où Néron est proclamé, il peut s'agir

\footnotetext{
${ }^{27}$ Tacite, Annales 12.69 .

${ }^{28}$ Il ne faut pas confondre fores Palatii et aditus domus, ce que fait C. Krause, 'Domus tiberiana', dans LTUR 2, 195

${ }^{29}$ La couronne navale étant proche d'un couronne civique, on a proposé d'identifier la domus à celle d'Auguste. Mais la couronne civique est devenue un symbole du pouvoir de ses successeurs ( $\mathrm{P}$. Zanker, Augusto e il potere delle immagini [Turin 1987], 100 ff.). Les Res Gestae 34 et les Fastes de Préneste (CIL I 2, 231) indiquent que la couronne augustéenne est suspendue super ianuam.

${ }^{30} \mathrm{Cf}$. J-P. Morel \& F. Villedieu, 'La Vigna Barberini à l'époque néronienne', dans Y. Perrin \& J.-M. Croisille, eds., Rome à l'époque néronienne, Neronia VI (Rome 1999), Latomus 2002, 74-96. Je laisse de côté la question des Adonaea.

${ }^{31}$ Cf. G. Morganti \& M.A. Tomei, 'Ancora sulla Via Nova', Mélanges de l'École Française de Rome, Antiquité 103 (1991) 2, 551-574.
} 
de marches latérales parallèlles à la voie, de gradins aménagés sur le clivus lui-même ou, solution la plus simple, du seuil des fores.

Entre 54 et 64, dans le cadre de la domus transitoria, Néron conserve l'héritage claudien. Lorsqu'il promet en 54 de séparer la gestion de sa cassette et celle de l'Etat et de ne plus enfermer la justice intra domum, c'est de la résidence claudienne qu'il s'agit. ${ }^{32} \mathrm{Si}$ on se fie à l'Octavie, le fastigium et la couronne navale doivent toujours être en place en 62 puisque la nourrice les évoque dans son monologue. ${ }^{33}$ Les innovations ne sont cependant pas mineures. La nourrice d'Octavie oppose la simplicité de la domus claudienne et les splendeurs clinquantes dont Néron couvre son aula. ${ }^{34}$ Le chantier de la domus tiberiana est ouvert ou poursuivi. Mais c'est l'association en un seul ensemble foncier du Palatium, de l'Esquilin et de l'ensellement qui les relie qui introduit la plus grande nouveauté sans cependant tout bouleverser puisque la domus transitoria n'est associée à aucun scandale. La domus familiale des Domitii, qu'il est plausible d'identifier dans les vestiges fossilisés sous le podium du temple de Vénus et de Rome, occupe une place centrale dans la nouvelle économie spatiale. Stricto sensu, la domus transitoria doit être cette domus qui assure le passage entre les deux collines. Aux demeures des Iulii et des Claudii - les deux pôles du pouvoir - Néron ajoute celle des Domitii, qui devient un troisième pôle. Le processus est révélateur de la nature d'un régime dont les titulaires restent attachés à leur famille. ${ }^{35}$

La nature de la nouvelle résidence, un ensemble de parcs, et l'affirmation de la nature monarchique du régime doivent être mises en relation avec le succès du mot aula dans la littérature contemporaine (cf. supra). Bien que les auteurs utilisent 'aula' dans un contexte littéraire en référence à des palais orientaux ou mythiques et que le terme appartienne à l'arsenal des loci communes servant à dénoncer le luxe dans lequel vit tout tyran, leurs descriptions constituent plus que des 'morceaux de poésie': elles ont quelque chose de familier; l' aula alexandrine de Lucain présente quelque communauté avec l'aula néronienne que l'auteur fréquente. Sans le nommer, Lucain (et Sénèque) ont en tête un complexe palatial qu'ils connaissent bien. ${ }^{36}$

\footnotetext{
${ }^{32}$ Tacite, Annales 13.4.

${ }^{33}$ Octavie 34-45.

${ }^{34}$ Octavie $624 \mathrm{ff}$.

${ }^{35}$ Néron reste fidèle à la domus familiale; plusieurs documents épigraphiques attestent les sacrifices qu'il y offre à la mémoire de son père.

${ }^{36}$ Aula évoque l'ensemble de la résidence et/ou le seul Palatium. Tacite, Annales 13.2, situe la manifestation qui se déroule en faveur d'Octavie en 62 in Palatio, l'auteur de l'Octavie dans l'aula
} 
Les accès à la résidence impériale subissent donc des changements difficiles à connaître. ${ }^{37}$ Entre 54 et 64 , domus aristocratiques privées et propriétés impériales continuent à voisiner, mais la définition même de la domus transitoria laisse supposer que Néron s'efforce d'assurer sa mainmise sur les domus situées entre sa maison familiale et le sommet du Palatin. Préfigurant celui de la domus aurea, l'accès à la domus transitoria associe vraisemblablement plusieurs entrées. Il est plausible d'imaginer une entrée générale au sommet de la pente qui monte du forum, à proximité de la domus familiale, voire dans cette domus. Ce n'est sans doute pas seulement en raison des contraintes du relief que seront aménagés ici le vestibule et les atria de la domus aurea, dont Suétone dit qu'elle est la domus transitoria restituta. L'accès à la colline du Palatin est marqué par des fores sur les 'clivus palatinus' (un peu plus au sud que l'arc de Domitien, à hauteur de la domus de la Vigna Barberini?) et/ou sur le 'clivus Victoriae '(permettant un accès direct à la domus palatina et à l' 'area palatina' par le prolongement du clivus). Dans un contexte encore républicain, la mise en scène du pouvoir n'est pas spectaculaire.

\section{4-70: domus aurea (plan II)}

L'incendie de 64 permet à Néron de réaménager l'espace en fonction des conceptions de la Neropolis et de la domus aurea. Les sources écrites décrivent les voies d'accès depuis le forum vers la domus aurea (urbanisme hippodamien, portiques, vestibule, atria, colosse) mais, uniquement sensibles aux innovations scandaleuses et peu aux continuités, elles observent un silence presque complet sur le Palatin et se taisent sur le chantier de la domus tiberiana. Un tel silence signifie que le Palatium conserve son rôle et que la nouvelle et pourtant colossale domus n'a rien de choquant. Les textes concernant les années 68-70 s'avèrent complémentaires: ils négligent la mise en scène des accès élaborée entre 64 et 68 , qui constituent pourtant le décor des guerres civiles, mais fournissent de précieux renseignements sur le Palatium et la domus tiberiana. La question des fores Palatii et des aditus domus se pose en termes inédits.

Il existe un large consensus pour voir dans le Palatin le pôle officiel de la domus aurea en opposition complémentaire avec l'édifice de l'Esquilin, considéré comme un pôle privé. Les réalités sont sans doute

(Octavie 780). Chez Sénèque aula $=$ palais $=\operatorname{arx}($ Agamemnon $78 \mathrm{ff} .$, Thyeste 697, 1078) . Chez Martial, Des spectacles 2.2 aula $=$ la domus aurea. Cf. n. 3.

${ }^{37}$ Cf. D. Palombi, Tra Palatino ed Esquilino Velia Cariae Fagutal (Rome 1997), 33-43. 
moins simples, mais peu importe ici. Quelques événements attestent le rôle politique unique de la colline, telles les statues de Tigellin et Nerva érigées in Palatio en remerciement de leur action dans la répression de la conjuration de Pison ${ }^{38}$ Le plus significatif est le triomphe de 68 qui s'achève non au Capitole, mais au temple d'Apollon Palatin. Les résonances augustéennes du lieu, récupéré et subverti, permettent l'affirmation d'un pouvoir à la fois légitime et original. Même dans sa variante néronisante, le Palatium demeure le lieu du pouvoir officiel. Les événements de 68-69 marquent la reconnaissance d'un régime monarchique désormais ouvert à tous en raison de l' extinction des familles qui l'ont fondé. Va de pair un consensus pour identifier dans le Palatium le lieu du pouvoir légitime détaché des familles de ses titulaires. ${ }^{39}$ Plutarque parle de l'hestia des Césars, Tacite de l'imperii arx, Dion Cassius du siège de l'archè. Il ne s'agit pas de métaphores. Le Palatium est le siège du pouvoir non seulement en raison de son aura sacrée et historique, mais aussi parce qu'y sont concentrées les instances techniques de la direction de l'empire (bureaux de la chancellerie, justice, armée, préfecture du prétoire) ${ }^{40}$ L'attitude des Flaviens le confirme $a$ contrario. ${ }^{41}$

On ne développera pas ici la question du vestibule, des atria et du colosse pour nous en tenir à la seule problématique des fores Palatii. Il est néanmoins indispensable de replacer la question dans le cadre des réaménagements de la pente qui mène du forum au Palatin. Les recherches récentes ont exclu les propylées spectaculaires imaginés par E. van Deman mais confirmé la nature hippodamienne du réaménagement et sa datation

\footnotetext{
${ }^{38}$ Tacite, Annales 15.72 .

${ }^{39}$ Galba, Othon et Vitellius s'y rendent dès leur proclamation et y résident. La fausse abdication de Vitellius en 69 fournit une information limpide: Vitellius sort du Palatium et songe à se réfugier dans sa domus familiale. Ses partisans s'y opposent - il ne saurait rentrer dans une domus privée qui ne peut être la résidence d'un prince - et l'obligent à regagner le Palatium. Ce retour enflamme la colère de l'adversaire: en rentrant dans une domus privée, Vitellius aurait montré qu' il était décidé à éviter ce qui pouvait donner l'idée du rang suprême; en regagnant le Palatium, il s'installe dans l'imperii arx (Tacite, Histoires 3.65-66).

${ }^{40} \mathrm{Cf}$. Y. Perrin, 'Imperii arx: métaphore ou réalité? Les fonctions de la 'domus tiberiana' néronienne', dans P. Defosse, ed., Mélanges C. Deroux III, Histoire et épigraphie, Droit, Latomus 270, 2003, p. 340-355. J'ai avancé l'hypothèse que la domus tiberiana était la concrétisation habile des promesses formulées en 54 de séparer la domus du prince et les affaires de l'Etat.

${ }^{41}$ Dans un souci de rupture, à la mort de Vitellius, Domitien ne se rend pas au Palatin, mais in paternos penatis et son père affecte de réduire l'importance du Palatium (cf. Josèphe, La guerre des Juifs 7.5; Tacite, Histoires 3.86 et Dion Cassius 65.10.4.; 66.10.4) En réalité, Vespasien considère bien que le Palatium est le lieu du pouvoir suprême; il en réinvestit la symbolique en laissant ostensiblement les thurias ouvertes et, en conserve, en les transformant, le vestibule, le colosse et la domus tiberiana.
} 
néronienne et montré son extension jusqu'au stagnum. Si les voies principales (via sacra et via nova) sont flanquées de portiques, les îlots abritent insulae et boutiques. Sur la pente qui descend vers le forum, l'articulation majeure du nouveau réseau viaire - via sacra est-ouest et voie nord-sud qui court désormais sur l'ensellement Palatin - Esquilin forme un T. A l' Est, s'étendent le podium des atria et les portiques du stagnum. La partie supérieure du $\mathrm{T}$ est identifiable au vestibule - à ciel ouvert - et c'est dans celui-ci (Suétone), sur la voie sacrée (Dion Cassius), devant les atria (Martial), donc au croisement des deux voies, que se dresse le colosse.

La création du parc de la domus aurea, les transformations du quartier entre forum, Esquilin et Palatin et l'achèvement de la domus tiberiana impliquent une modification des limites de la résidence impériale dont l'extension fait scandale, et donc des changements dans la topographie des fores Palatii. La nouvelle organisation de l'espace suggère que le complexe de la domus aurea possède plusieurs entrées et que plusieurs portes peuvent se succéder sur le même itinéraire, à l'instar de l'organisation des accès à l' arx de Domitien telle que l'évoque Pline (cf. supra). Le vestibule joue un rôle essentiel: architecturalement, il assure l'articulation des deux ensembles de constructions couvrant les pentes vers le forum et le stagnum en cachant la légère différence de leurs orientations. ${ }^{42} \mathrm{~A}$ son niveau s'ouvrent deux - voire trois - voies distinctes, vers le stagnum et le parc de part et d'autre du podium des atria, vers le Palatin par le 'clivus palatinus' (et vers l'Esquilin au nord?). Que le statut juridique du vestibule soit public ou privé, qu'il soit 'dedans' ou 'dehors', sa fonction est d'assurer la transition entre Rome et la résidence impériale. On peut donc rechercher les fores Palatii soit à son entrée, soit là où on le quitte pour pénétrer véritablement dans la domus du prince.

Le lieu où le 'clivus palatinus' débouche sur 1' 'area palatina' constitue un noeud de l'économie spatiale des aménagements. A son niveau s'achèvent le clivus désormais rectiligne et ses portiques qui s'articulent avec le vestibule et l'ensemble qui couvre les pentes descendant vers le forum et le stagnum. ${ }^{43}$ Au sud s'étendent l' 'area palatina' au fond de laquelle s'élève une aula dont l'existence après 64 est assurée et, au delà, un quartier dont les

\footnotetext{
${ }^{42}$ D'où sans doute le plan trapézoïdal partiellement mis à jour; cf. M. Medri, 'Suetonius, Nero 31,1: elementi et proposte', dans Cl. Panella, ed., Meta Sudans (Rome 1996), 175-177.

${ }^{43}$ Le réaménagement du clivus est néronien. Cf. les contributions du Bullettino della Commissione Archeologica Comunale in Roma 91 (1986): M.A. Tomei, 413-414 et 518, R. Santangeli et R. Volpe 418-419, G. Morganti, 426; voir aussi Archeologia Laziale 8 (1987), 79.
} 
travaux de A. Cassatella laissent soupçonner l'importance. ${ }^{44}$ Le grand bloc de maçonnerie de datation incertaine qui se trouve dans l'alignement des portiques pourrait être non les substructions d'un temple (aedes Iovis Victoris?), mais celles d'un édifice articulé avec les fores et surplombant scénographiquement l'accès au Palatin. ${ }^{45}$ A l'Ouest, on accède à la domus tiberiana par une branche du cryptoportique oriental orientée est-ouest, qui s'ouvre à sa hauteur. ${ }^{46} \mathrm{~A}$ l'Est, l'existence de la domus de la Vigna Barberini entre 64 et 68 est hypothétique. ${ }^{47}$ L'existence d'une entrée à cet emplacement n'est guère douteuse. Situer des fores néroniennes au niveau du site occupé par l'arc de Domitien est plausible. ${ }^{48}$ Les gradus seraient alors les marches qui donnent accès à l'édifice sur podium que certains identifient à l'aedes Iovis ou celles qui relient les niveaux de la voie et du portique latéral, ou, simplement, les gradins qui marquent le seuil de la porte.

Mais si on considère que les fores constituent l'entrée de l'aire impériale, il convient de les chercher plus bas, devant le vestibule. Il conviendrait donc de les situer sur le tronçon le plus élevé de la voie sacrée, devant le colosse. On émettra l'hypothèse que l'arcus in summa sacra via du relief des Haterii n'est autre que cette porte transformée par Vespasien. ${ }^{49}$ Les gradus seraient alors des gradins qui marquent le seuil de l'arc. Dans ce cas de figure, il faut imaginer un autre poste de contrôle au sommet de la nova via. Les deux propositions topographiques ne sont pas exclusives: comme l'indique Pline le Jeune pour l'époque de Domitien, plusieurs fores et postes de garde peuvent se succèder sur la voie sacrée et le 'clivus palatinus' (et sur les voies qui descendent vers le stagnum.). ${ }^{50}$

Sur le chemin du Palatin au forum par le 'clivus Victoriae' et les escaliers qui descendent vers la fontaine de Juturne ou les aménagements de

\footnotetext{
${ }^{44}$ Cf. A. Cassatella, 'Domus Aurea: complesso del Palatino', dans LTUR 2, 63.

${ }^{45}$ Notre proposition semble rejoindre celle de M.A. Tomei, dans Bullettino della Commissione Archeologica Comunale in Roma 91 (1986), 518.

${ }^{46} \mathrm{P}$. Romanelli, 'Il criptoportico del Palatino', dans Les cryptoportiques dans l'architecture romaine, Coll. de l' École Française de Rome (Rome 1973), 207.

${ }^{47}$ Cf supra, p. 364 n. 30.

${ }^{48}$ Sur la chronologie de l'arc de Domitien Cf. A. Cassatella, 'Arcus Domitiani', dans LTUR 1, 92.

${ }^{49}$ Dans cet arc, F. Coarelli, Il Foro Romano I (Rome 1983), 232 ff. voit une représentation de la porte Mugonia reconstruite après 64 et M. Torelli, Typology and structure of roman historical Reliefs (Ann Arbor 1982), 134 l'arc de Vespasien.

${ }^{50}$ Quid de l'arc quadrifrons proche de l'arc de Titus daté d'après 64 (cf. J. Arce \& R. Mar, 'Monumento presso l'arco di Tito nel foro Romano', Archeologia Laziale 10 [1990], 43 ff.)? Lorsqu'il apprend la défection d'une partie de ses troupes, Vitellius descend du Palatium pour se rendre au forum; ses partisans lui imposent de regagner le siège du pouvoir en reprenant le chemin qu'il avait parcouru, la Via Sacra et donc le 'clivus palatinus' (Tacite, Histoires 3.67-68, 70, 84).
} 
1' atrium de Caligula (dont on ignore l'état entre 64 et 68), on attend la présence de fores et d'un poste de garde sur le clivus, près du sommet de l'escalier, là où se trouvait l'antique porta Romanula. On peut éventuellement en voir la trace dans les médiocres vestiges qui gisent là de part et d'autre du clivus. ${ }^{51}$ Très proches des aditus domus, ces fores donnent accès à la domus tiberiana autant qu'au Palatin: les ayant franchies, le visiteur peut accéder soit à la domus par son aditus central (cf. ci-dessous), soit à l' 'area palatina' par le prolongement du 'clivus Victoriae'. Lorsque sous Galba les chevaliers et les sénateurs, puis sous Othon les soldats vitelliens forcent les fores Palatii pour rencontrer les deux princes, Tacite donne le sentiment qu'ils pénètrent directement dans la domus tiberiana. On peut situer la scène ici. Les gradus Palatii seraient alors les scalae graecae et/ou annulariae qui descendent vers le forum... ou le seuil de la porte.

Quant aux aditus et gradus domus, il ne peut s'agir que de ceux de la domus tiberiana. sinon achevée, du moins fonctionnelle en $68 .^{52} \mathrm{Si}$ son architecture et son répertoire décoratif sont à peu près inconnus, les données planimétriques révèlent un plan unitaire prétabli rigoureusement géométrique. La domus proprement dite s'élève au centre du podium et est environnée par des jardins. ${ }^{53}$

Par son plan centré, la construction s'inscrit dans la culture de l'architecture domestique à atrium central, mais à contre-courant de l'évolution contemporaine de celle-ci qui développe des plans en longueur. Son site et sa surélévation l'inscrivent dans un espace urbain prestigieux. ${ }^{54}$ L'ampleur de ses proportions et son plan axialisé sont proches de ceux des grands édifices publics contemporains (macellum, thermes). Le niveau noble s'étendant sur la plate-forme du podium, des escaliers sont nécessaires pour l'atteindre et les façades sont aménagées sur deux niveaux au moins. L'organisation symétrique du podium ne désigne aucune des façades comme

\footnotetext{
${ }^{51}$ E. Wadsworth, Memoirs of the American Academy in Rome, 4 (1924), 44-45 signale des vestiges néroniens sur le côté méridional. Sur les constructions antérieures à l' avant-corps nord-occidental domitianéen de la domus tiberiana, cf. C. Krause, Domus Tiberiana (Zurich 1985), 89 ff., fig. 94, 112, $116,117,121 \mathrm{ff} ., 131 \mathrm{ff}$.

${ }^{52}$ Néron inaugure la domus aurea (Suétone, Néron 31), ce qui veut dire que ses deux pôles de l'Esquilin et du Palatin étaient considérés comme achevés. On peut penser que la domus a été inaugurée en 66 , sommet du règne marqué par la venue de Tiridate et le départ pour la Grèce en septembre. La longue absence programmée du prince incite à penser qu'à son départ le chantier du Palatin était à son terme.

${ }^{53}$ C. Krause, op.cit. (n. 28), 193

${ }^{54}$ Cf. l'opposition soulignée par H. Drerup, 'Bildraum und Realraum', Rheinisches Museum 1959, 173 entre espace intérieur enchassé de l'architecture privée et espace illimité des constructions publiques et impériales.
} 
plus importantes que les autres; la restitution de quatre façades identiques en élévation constitue l'hypothèse la plus simple. C'est celle que retient $\mathrm{C}$. Krause qui restitue un portique sur les quatre faces externes, qui nous intéressent ici.

Le réseau des corridors qui structurent le podium le découpe en neuf blocs rectangulaires. La tradition romaine établissant une adéquation organique et morphologique entre la structure interne des édifices et les rythmes fondamentaux de ses motifs architecturaux, on peut exclure que l'organisation des façades soit sans rapport avec eux. Chacun des côtés de la résidence repose sur la juxtaposition de trois massifs de substructions, ce qui suggère que l'architecte a donné à ses façades un rythme ternaire. Le centre de chacune des façades est le lieu où l'on attend un aditus et des gradus. Cette restitution correspond au schéma de la scaenae frons. dont les valeurs royales ont, depuis l'époque hellénistique, inspiré l'architecture italienne commanditée par les imperatores. Alexandrie constitue sans doute l'archétype, mais Vergina fournit la seule comparaison documentée. Le palais macédonien et la domus tiberiana ont en commun leur plan centré et leur surélévation sur une terrasse. ${ }^{55}$ Ils jouent le même rôle politique éminent. Il y a des chances pour que la façade de la batisse romaine soit du même type que celle du palais macédonien: celle-ci est précédée par un portique et articule deux 'ailes' qui encadrent un module central occupé par l'entrée aulique surmontée par un fronton. ${ }^{56} \mathrm{La}$ domus néronienne se substituant à celle de Claude et abritant le coeur du pouvoir, il y a des chances pour qu'elle en reprenne la symbolique et qu'un fastigium surmonte la (les ?) façade(s). ${ }^{57}$

La façade occidentale de la domus tiberiana est inconnue. Son front méridional est précédé par des salles postérieures à 64 . Assez étonnante est la présence de telles salles, quelles que soient leurs fonctions (garde, bureaux, magasins), sur une façade qui domine le prestigieux quartier de la domus d'Auguste, des temples de Cybèle et de la Victoire. Dans tous les cas, la mise en scène ne semble pas exploiter l'aura du lieu. La façade orientale

\footnotetext{
${ }^{55}$ Le thème de la scaenae frons est à l'ordre du jour dans les années 60-70. Sur les palais hellénistiques et notamment Vergina, cf. I. Nielsen, Hellenistic Palaces (Aarhus 1999), 81 ff. et ma contribution aux Mélanges C. Deroux, citée n. 40.

${ }^{56}$ Cf. I. Nielsen, op. cit. (n. 55), et G. Brands, Halle, Propylon und Peristyl, dans Basileia. Die Paläste der hellenistischen Könige (Mayence 1996), 64 ff.

${ }^{57}$ Bien que la domus tiberiana ait connu de nombreuses modifications entre Néron et Antonin, on en verra un indice dans un passage de l'Histoire Auguste qui concerne l'installation de Marc Aurèle par Antonin dans la domus en 140: aulico fastigio renintentem ornavit (SHA, Marcus 6.3).
} 
domine 1' 'area palatina' que traverse le visiteur qui se rend à la domus tiberiana. C'est là qu'il faut situer le discours de Pison à la cohorte des prétoriens. L'architectonique de la domus et les nécessités fonctionnelles et symboliques du cheminement veulent que des gradus se situent au centre de la façade, éventuellement dominé par un fastigium. Visible depuis le forum et la Velia, la façade septentrionale occupe une situation privilégiée dans le paysage urbain. On peut penser qu'elle a reçu un traitement spécifique servant la mise en scène du pouvoir comme c'est le cas à l'époque de Domitien. De fait, les aménagements du Flavien ont été précédés par d'importants escaliers. ${ }^{58}$ On y reconnaîtra les gradus domus proches des fores Palatii du 'clivus Victoriae' évoqués plus haut.

\section{Conclusions}

La connaissance des accès au Palatin et à la domus tiberiana entre 54 et 70 conserve bien des zones d'ombre. L'étude montre la spécificité du Palatin au sein de la domus aurea. Le parc et la fabrique de l'Esquilin peuvent être considérés comme l'expression et le coeur de ce qu'il est convenu d'appeler le 'néronisme'. Les aménagements de la pente qui donne accès aux deux pôles (Esquilin et Palatin) depuis le forum, le vestibule, les atria et le colosse qui domine le paysage exaltent Néron. On retrouve certains traits de son axiologie sur le Palatium (cenatio, triomphe de 68). Mais la domus aurea conserve au Palatium son rôle unique dans la vie politique et le paysage de l'Urbs. Même dans sa variante néronisante, il demeure le lieu officiel du pouvoir suprême. C'est le seul legs que cultivent les successeurs du prince honni dès sa mort en 68 .

L'oeuvre de Néron rend définitif le redéplacement amorcé dès Tibère du centre de gravité du Palatium, de l'aire augustéenne méridionale vers l'aire septentrionale. Pour un moderne qui déchiffre le paysage, la domus tiberiana exprime topographiquement et architecturalement la nature personnelle du pouvoir qui domine politiquement la Res Publica et ce qui était son coeur, le forum. Or aucun auteur ancien n'établit ce rapprochement entre topographie et politique. C'est sans doute que la sensibilité des anciens diffère de la notre, mais c'est aussi parce que la mise en scène du pouvoir palatin n'est pas scandaleuse. La domus tiberiana est la construction d'un homme d'Etat, non celle d'un artiste. Elle s'inscrit dans une culture politique qui ne doit rien aux valeurs solaires et agonales qui sont censées être au coeur de l'expérience néronienne. Quant au Palatium, il est sans doute clos

${ }^{58}$ Krause, op.cit. (n. 28), 89 ff., 116-117, 124-125, 134. 
puisque des fores en définissent l'accès et que plusieurs postes de garde s' échelonnent sur les voies qui mènent aux lieux où le prince gouverne ${ }^{59} \mathrm{Y}$ accéder suppose qu'on a gravi la pente montant du forum, traversé le vestibule et ressenti la puissance du prince dont le portrait colossal domine tout. Mais la mise en scène des accès au Palatin proprement dit et de la domus tiberiana empruntent ses motifs à un répertoire romain connu depuis la fin de la république et Auguste. Par leur site, les fores semblent renvoyer aux portes archaïques (Mugonia, Romanula et Romana sur le 'clivus Victoriae $^{, 60}$ ) et identifier le Palatium néronien au Palatium romuléen. Cette inscription dans l'histoire romaine et la défnition topographique, architecturale et fonctionnelle du cœur de l'empire permettent de rendre compte du rôle joué par le Palatium dans l'histoire de l'empire dès 68 alors que le reste de la domus aurea est abandonné.

Lyon, août 2002

\footnotetext{
${ }^{59}$ Aucune source ne signale l' existence d' une telle cloture. Dès Caligula, il est possible de 'boucler' le Palatium (Suetone, Caligula 14). La question de la clôture du parc de la domus aurea demeure posée.

${ }^{60}$ F. Coarelli 1983, op.cit. (n. 49), 228, 231 ff. L'existence de fores ici est probable, mais les sources n'ont pas l' occasion de les mentionner.
} 


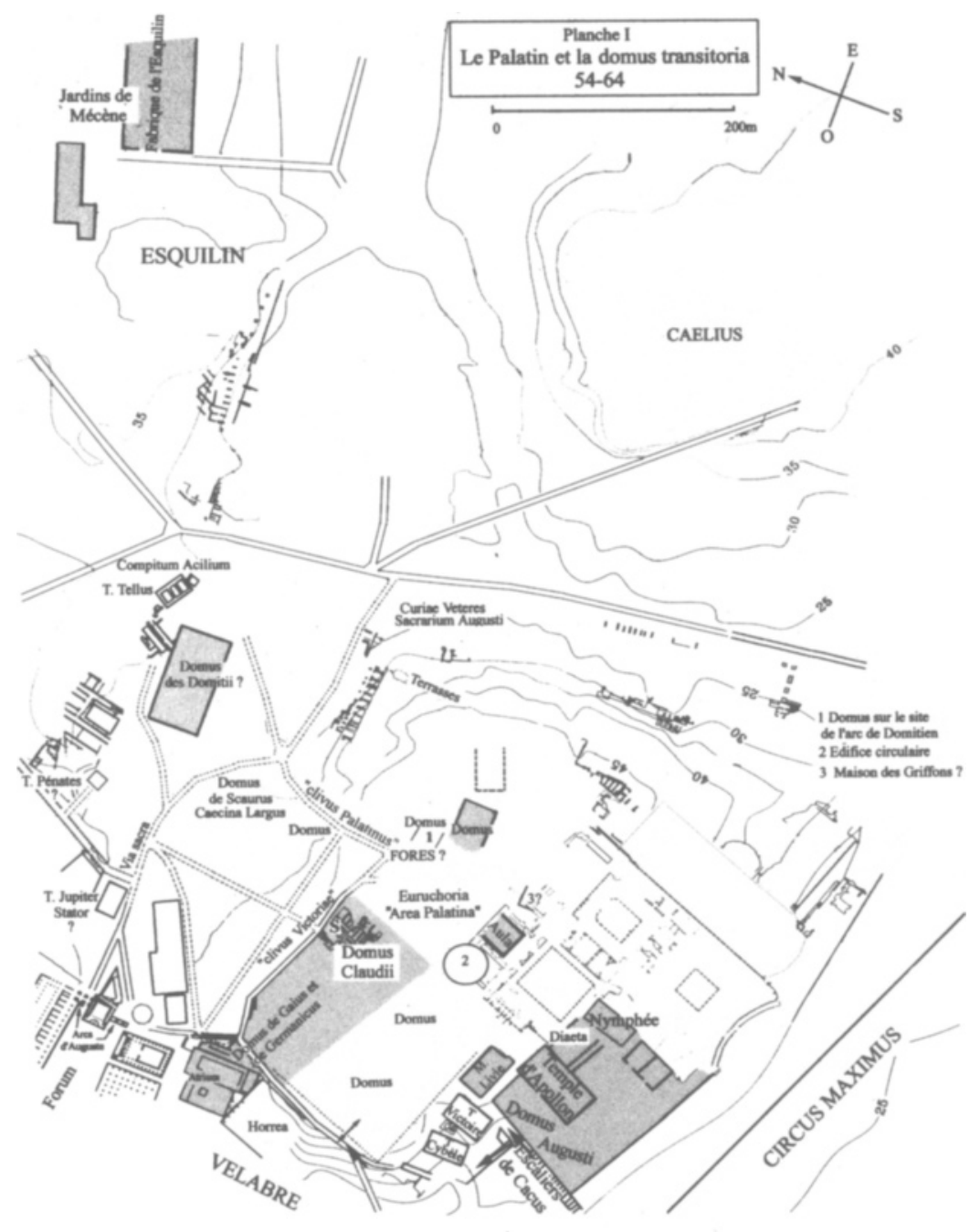

YVES PERRIN - 9789004401631 


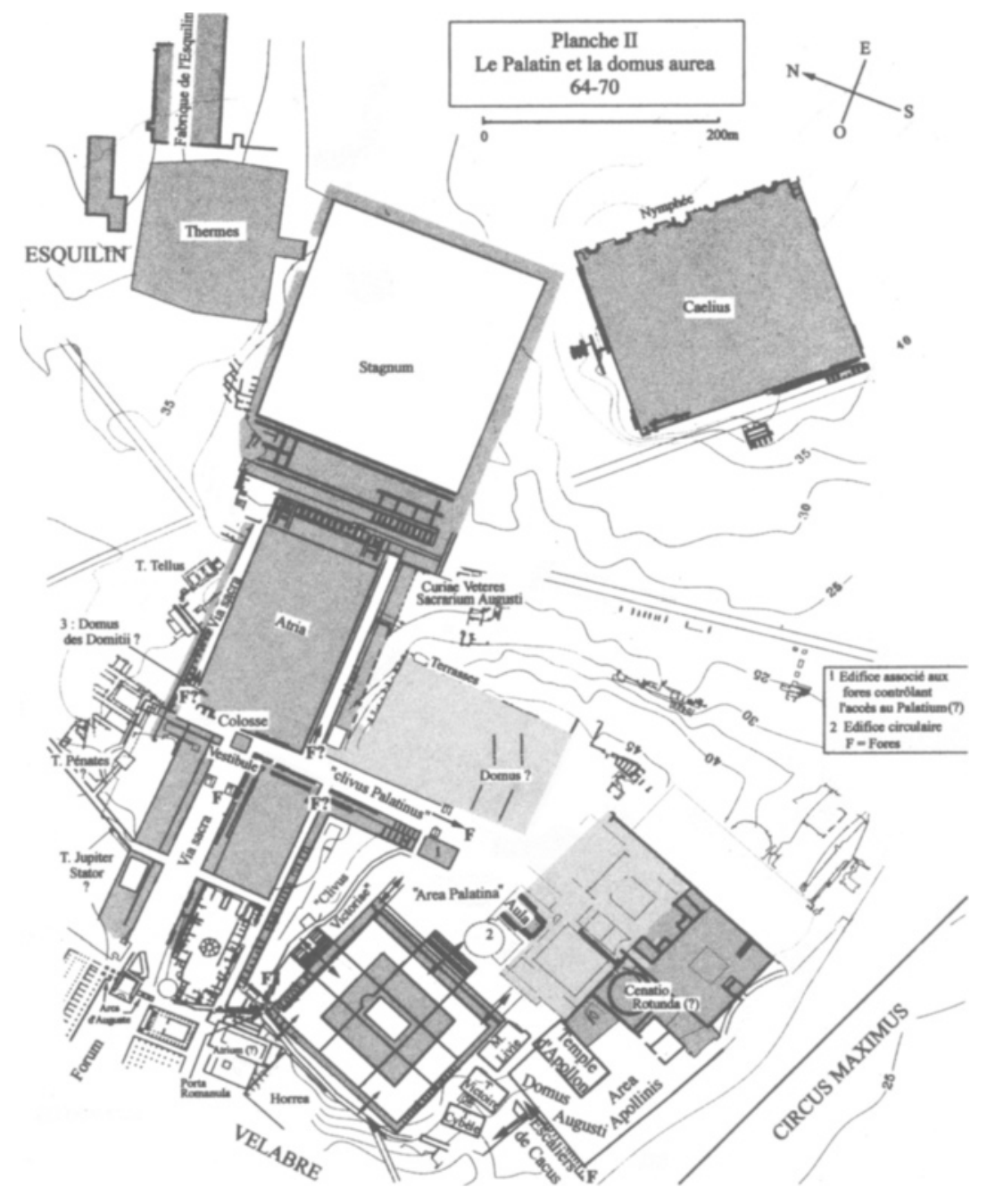

